\title{
Notch Signaling in the Astroglial Phenotype: Relevance to Glutamatergic Transmission
}

\author{
López-Bayghen Esther, Angulo-Rojo Carla, \\ López-Bayghen Bruno, \\ Hernández-Melchor Dinorah, Ramírez Leticia and \\ Ortega Arturo
}

Additional information is available at the end of the chapter

http://dx.doi.org/10.5772/intechopen.73318

\begin{abstract}
Glutamate (Glu), the major excitatory neurotransmitter, elicits its action through the activation of membrane receptors and transporters expressed in neurons and glial cells. Glial glutamate transporters, EAAT1 and EAAT2, remove this transmitter from the synaptic cleft preventing an excitotoxic insult. The Notch pathway is a signaling system involved in neuro- and gliogenesis. Radial glia (RG) generates neurons, oligodendrocytes, and astrocytes in a spatial and temporal pattern, in which Notch represses neurogenesis, maintaining the self-renewal potential of RG. Astrogenesis depends on several stimuli, Notch being a master regulator of the differentiation process. The cAMP-PKA-CREB signaling cascade cross talks with the Notch pathway, acting synergistically by reducing progenitor markers and inducing astrocytic differentiation. Notch1 mRNA is upregulated in a PKA $/ \gamma$-secretase/NICD/CSL-dependent manner, suggesting a feedback loop to keep Notch active until astrocytic differentiation is complete. Glial differentiation is also modulated by PKC, which acts over NICD. In RG cells and astrocytes enwrapping glutamatergic synapses, EAAT1 transcriptional regulation is mediated by PKC, increasing Notch expression and its receptor intracellular traffic. It is clear that Notch represents an activity-dependent molecular key in RG cells that enable them to shape glutamatergic transmission through the expression of genes involved in glial/neuronal interactions.
\end{abstract}

Keywords: glia cells, signal transduction, Notch, differentiation, protein kinase C

\section{Introduction}

Glutamate (Glu), the major excitatory neurotransmitter in the central nervous system (CNS), is a key player in higher brain functions such as learning and memory, and it is also 
involved in cell differentiation and synaptogenesis. Glu exerts its function through specific receptors, according to which the signal transduction pathway mechanisms are classified into two major groups: ionotropic (iGluRs) and metabotropic receptors (mGluRs). iGluRs are ligand-gated ion channels subdivided upon pharmacological and electrophysiological properties into NMDA, AMPA, and KA receptors. mGluRs are G protein-coupled receptors subdivided in accordance with their amino acidic sequence and pharmacological properties into three subgroups, preferentially activated by quisqualate (Quis), t-ACPD, and L-AP4, respectively [1-4].

Cerebellar Bergmann glia cells (BGC) are radial glia (RG) cells that are not differentiated into astrocytes after birth [5] and function as a neuronal reservoir [6, 7]. These cells extend processes through the molecular layer completely surrounding excitatory synapses between Purkinje cells and both parallel and climbing fibers. An exquisite and complex interplay between presynaptic-postsynaptic neurons and glia cells is fundamental for glutamatergic transmission. Glu recycling depends upon these interactions. Glu is removed from the synaptic cleft by a family of electrogenic sodium-dependent transporters expressed in neurons and glia cells [8]. Five subtypes of transporters named excitatory amino acid transporters 1-5 (EAAT1-5) have been characterized. The glial transporters EAAT-1 (GLAST) and EAAT-2 (GLT-1) account for more than $80 \%$ of the Glu uptake activity in the brain $[9,10]$. Within BGC, EAAT-1/GLAST is the predominant transporter [11].

Once internalized, Glu is metabolized to Gln via Gln synthetase and released in the vicinity of the presynaptic neuron through sodium-dependent neutral amino acid transporter (SNAT) 3. Gln is then taken up by the presynaptic neuron through SNAT 2 and converted back to Glu by the enzyme glutaminase to be packed into synaptic vesicles completing the so-called Glu/ Gln shuttle (reviewed in [12]). It is this kind of glial/neuronal interactions that gave rise to what has been known in the last years as a tripartite synapse [13]. Evidence suggests that Glu transporters might also participate in the signaling transactions triggered by this excitatory amino acid. In fact, Glu regulates the uptake process in a receptor-independent manner [14]. More recently, it has also been reported that EAAT- 1 is coupled to the $\mathrm{Na}^{+} / \mathrm{K}^{+}$ATPase $[15,16]$ and to the Gln transporter SNAT3 [17].

In this context, we reviewed in this contribution the role of Notch signaling in RG focusing in its role in EAAT-1/GLAST regulation as a key element in the molecular mechanisms that support the proven glia contribution to glutamatergic neurotransmission.

\section{Glutamatergic transmission: role of glial cells}

Glutamate (Glu) is the major excitatory neurotransmitter in the vertebrate brain. It elicits its action through the activation of specific membrane receptors and transporters expressed both in neurons and in glial cells. Extracellular glutamate levels have to be tightly regulated in order to prevent Glu receptors over-stimulation that has been shown to result in neuronal death, phenomena commonly known as excitotoxicity. A family of sodium-dependent Glu 
transporters particularly enriched in glial cells is responsible for the removal of this transmitter from the synaptic cleft [12]. These transporters, known as excitatory amino acid transporters (EAAT), are differentially expressed in neurons and astrocytes. EAAT3, 4, and 5 are mainly neuronal, whereas EAAT1 and EAAT2 are glial, although the latter one has also been found to be present in certain neuronal populations [18]. Once Glu has been taken up by glial cells, it is mostly converted to glutamine (Gln) by the glial-expressed Gln synthetase to be released in the vicinity of the presynaptic terminal, a process known as the Glu/Gln shuttle, in which an exquisite interplay between neurons and glial cells is fundamental for the proper function of glutamatergic transmission [12]. In this context, glutamatergic synapses are a perfect example of what has been lately known as a tripartite synapse [19].

\section{Notch signaling}

Notch signaling involves cell to cell communication and has a simple core. It initiates when the Notch receptor (Notch 1-4), present in the receiving-signal cell, binds its ligand (Jagged/Delta-like) present in the sending-signal cell. This binding promotes two sequential proteolytic cleavages on the Notch receptor: the first is mediated by the protease ADAM10/TACE (tumor necrosis factor $\alpha$ converting enzyme) to generate the membranetethered intermediated Notch extracellular truncation (NEXT). The second cleavage is mediated by the $\gamma$-Secretase enzyme on NEXT, to release the signal effector Notch intracellular domain (NICD) into the cytoplasm. NICD is translocated to the nucleus where it binds the transcription factor CSL (CBF1/RBPJ $\kappa$ in vertebrates, suppressor of hairless in Drosophila, Lag-1 in C. elegans) to activate what is known as the canonical Notch pathway. In the absence of NICD, CSL associates with the ubiquitous co-repressors (Co-R): SKIP, CtBP/Hairless, SMRT, CIR, FLH1C/KyoT2, SHARP/MINT and Gro/TLE proteins, and histone deacetylases (HDACs) to halt the transcription of Notch target genes. Once NICD binds CSL, allosteric changes may occur on CSL that facilitates displacement of transcriptional repressors. The transcriptional co-activator Mastermind (MAM) then recognizes the NICD/CSL interface, and this tri-protein complex recruits additional co-activators (CoA) to promote transcription of target genes, as the astroglial markers shown in Table 1 [20-27].

The noncanonical Notch signaling pathway is CSL-independent and can as well be either ligand-dependent or independent. Nevertheless, one has to keep in mind that the NICD/ CSL complex is the major effector of Notch signaling. Several pieces of evidence have demonstrated that the Notch pathway may signal independently of CSL. It was first reported that Notch could signal via the RING-domain of E3 ubiquitin ligase Deltex1 (DTX1) [28]. It has also been shown that NEXT binds NICD on its ankyrin repeats [29], leading to its nuclear translocation. It has been documented as well that the NICD/DTX1 complex interacts with the transcriptional co-activator p300 inhibiting the transcriptional activation of the neural-specific transcription factor MASH1 [30]. As it will be described later, other genes important for astroglial differentiation are also targets of NICD/DTX (Table 1). 


\begin{tabular}{|c|c|c|c|}
\hline & Target & Function & Reference \\
\hline \multirow[t]{5}{*}{$\begin{array}{l}\text { Canonical pathway } \\
\text { (NICD/ } \\
\text { RBPJא-dependent) }\end{array}$} & Hes1/Hes5 & $\begin{array}{l}\text { Down-regulate pro-neural transcription factors, } \\
\text { as Mash1, Math, and Neurogenin; which in turns } \\
\text { regulate neural protein expression (p. ej.MAP2) }\end{array}$ & {$[72]$} \\
\hline & $\begin{array}{l}\text { Glutamate aspartate } \\
\text { transporter (GLAST) }\end{array}$ & Glutamate transport & This work \\
\hline & $\begin{array}{l}\text { (Glial fibrillary acidic } \\
\text { protein (GFAP) }\end{array}$ & $\begin{array}{l}\text { Principal protein (most abundant) forming an } \\
\text { intermediate filament in mature astrocytes. Is } \\
\text { important in radial glia cytoskeleton. }\end{array}$ & {$[50]$} \\
\hline & $\begin{array}{l}\text { Binding lipid-binding } \\
\text { protein (BLBP) }\end{array}$ & $\begin{array}{l}\text { Hydrophobic protein member from the family FABP } \\
\text { (Fatty acid-binding protein). Binds to ligands of } \\
\text { nuclear receptors and participate regulating their } \\
\text { transcriptional activity. }\end{array}$ & {$[32,33]$} \\
\hline & Vimentin & $\begin{array}{l}\text { Most abundant protein forming intermediate } \\
\text { filaments in immature astrocytes and radial glia }\end{array}$ & [73] \\
\hline \multirow{4}{*}{$\begin{array}{l}\text { Noncanonical } \\
\text { (NICD/ } \\
\text { RBPJא-independent) }\end{array}$} & erbB2 & Tyrosine kinase receptor & [33] \\
\hline & Slug & $\begin{array}{l}\text { Zinc-finger transcription factor that regulates neural } \\
\text { crest formation and delamination }\end{array}$ & [74] \\
\hline & $\begin{array}{l}\beta \text {-catenin/Wnt } \\
\text { signaling }\end{array}$ & $\begin{array}{l}\text { Wnt/ } \beta \text {-catenin signaling; Notch binds and titrates } \\
\text { levels of the obligate Wnt-signaling component active } \\
\beta \text {-catenin. }\end{array}$ & {$[75,76]$} \\
\hline & $\mathrm{BMP} 4$ & $\begin{array}{l}\text { Induce neural crest cells from the neural plate. Bmp4 } \\
\text { can induce Slug expression and subsequent neural crest }\end{array}$ & {$[74,77]$} \\
\hline
\end{tabular}

Table 1. Targets of Notch signaling pathway in central nervous system development.

\section{Notch pathway signaling in astroglial differentiation}

Notch pathway is a pivotal signaling system during neuro- and gliogenesis in the central nervous system (CNS) [24, 25]. Primary neural stem cells (NSC) are radial glia (RG) during development, characterized by the expression of astroglial markers such as the astrocytespecific glutamate/aspartate transporter (GLAST), the brain lipid-binding protein (BLBP), and tenascin C (TN-C) [31]. RG cells generate neurons, oligodendrocytes, and astrocytes in a characteristic spatial and temporal pattern [31]. In this context, the Notch pathway plays an essential role repressing neurogenesis and maintaining the self-renewal potential of RG.

On the neurogenic phase, RG divide asymmetrically for auto-renewal and generation of neurons or neuron-restricted intermediate progenitor cells (nIPCs, transit amplifying cells), which in turn populate the subventricular zone (SVZ) in the cortex. The newborn neurons migrate along parental RG fibers, even though RG are dividing [31]. The Notch pathway plays an important role during the neurogenic phase in several ways. In the cortex, recent findings suggest that Notch signaling among SVZ nIPCs and between nIPCs and RG is important in the regulation of progenitor proliferation and in the inhibition of precocious neuronal differentiation. RG receives Notch signaling to activate Hes1 and Hes5 transcription factors, which down-regulate pro-neuronal genes such as neurogenin 1 (Ngn1), Mash1, and Math. 
At the same time, Ngn1 becomes an astrogenesis inhibitor through the sequestration of p300/ CBP, a key inducer of astrocyte differentiation [29].

In the cerebellum and in the immature RG, it has been demonstrated that Notch1 is activated by Jagged1 on newborn neuron progenitors; this interaction regulates the molecular and morphological differentiation of RG, through the transcriptional activation of BLBP and the erbB2 receptor tyrosine kinase. This effect is mediated by two downstream mechanisms, one that depends on RBPJ $\kappa$ (canonical activation) and the other depending on Deltex1 (DTX1) (noncanonical activation). In this manner, the induced erbB2 receptor interacts with its ligand neuregulin, present on neuronal progenitors, to facilitate cell migration through RG fibers (Figure 1) [32, 33].

After the neurogenesis period, at the end of embryonic development, most of the RG cells have lost their ventricular attachment and migrate toward the cortical plate by a process of somal translocation. In mammals, the majority of RG cells are transformed into astrocytes. During this period, astrocytic and oligodendrocytic intermediate precursors are also generated (aIPCs and oIPCs). Some studies suggest the presence of multipotent and bipotent progenitors, and perhaps astrocyte-restricted progenitors in the neonatal SVZ [34].

Astrogenesis depends on several stimuli, being the Notch pathway a master regulator of the differentiation process. During the gliogenic phase, RG progenitors gain competence to generate astrocytes due to the activity of growth factors such as basic fibroblast growth factor (bFGF) and epidermal growth factor (EGF). This gain of competence allows them to respond to specific gliogenic signals acting at the extracellular level to activate astrocyte markers such as glial fibrillary acidic protein (GFAP), S100 $\beta$, aquaporin 4, glutamate transporters (GLT-1, EACC1, and GLAST), and aldehyde dehydrogenase 1 family, member L1 (AldhL1) [35-39]. Before the astrocyte-marker promoters can respond to gliogenic signals, a chromatin epigenetic remodeling must occur. Notch canonical activation on RG induces expression of nuclear factor 1A (NFA1), an inhibitor of the DNA methyltransferase 1 (DNMT1). DNMT1 keeps STAT3 site of GFAP promoter methylated and inactive [40, 41].

The extracellular signals are provided by neurotrophic cytokines such as ciliary neurotrophic factor (CNTF), leukemia inhibitory factor (LIF), and cardiotrophin-1 (CT-1) secreted by newborn neurons. These cytokines activate heterodimeric cell surface receptors composed of two subunits named LIFR $\beta$ and gp130, which in turn activate to members of the JAK family of tyrosine kinases that result in the phosphorylation and nuclear translocation of signal transducer and activator of transcription (STAT) proteins. In RG, two of these proteins, STAT1 and STAT3, act on specific sites in the promoters of the astroglial genes GFAP and S100 $\beta$ to stimulate their transcription during the astrocyte differentiation process. Neural progenitors also respond to different neurotrophic factors from the bone morphogenetic proteins (BMP) family to generate astrocytes. In this case, BMP2 and BMP4 act on heterotrimeric receptors, which activate SMAD transcription factors. These, in turn, interact with activated STAT proteins to synergistically stimulate transcription of glial-specific genes during astrocyte differentiation [42-47].

Another estrogen signal is the activation of the seven transmembrane domain G protein-coupled receptors by the pituitary adenylate cyclase-activating polypeptide (PACAP), triggering the differentiation of astrocytes by increasing intracellular cAMP and activating the cAMPdependent protein kinase (PKA), which translocates into the nucleus to phosphorylate and 
A)
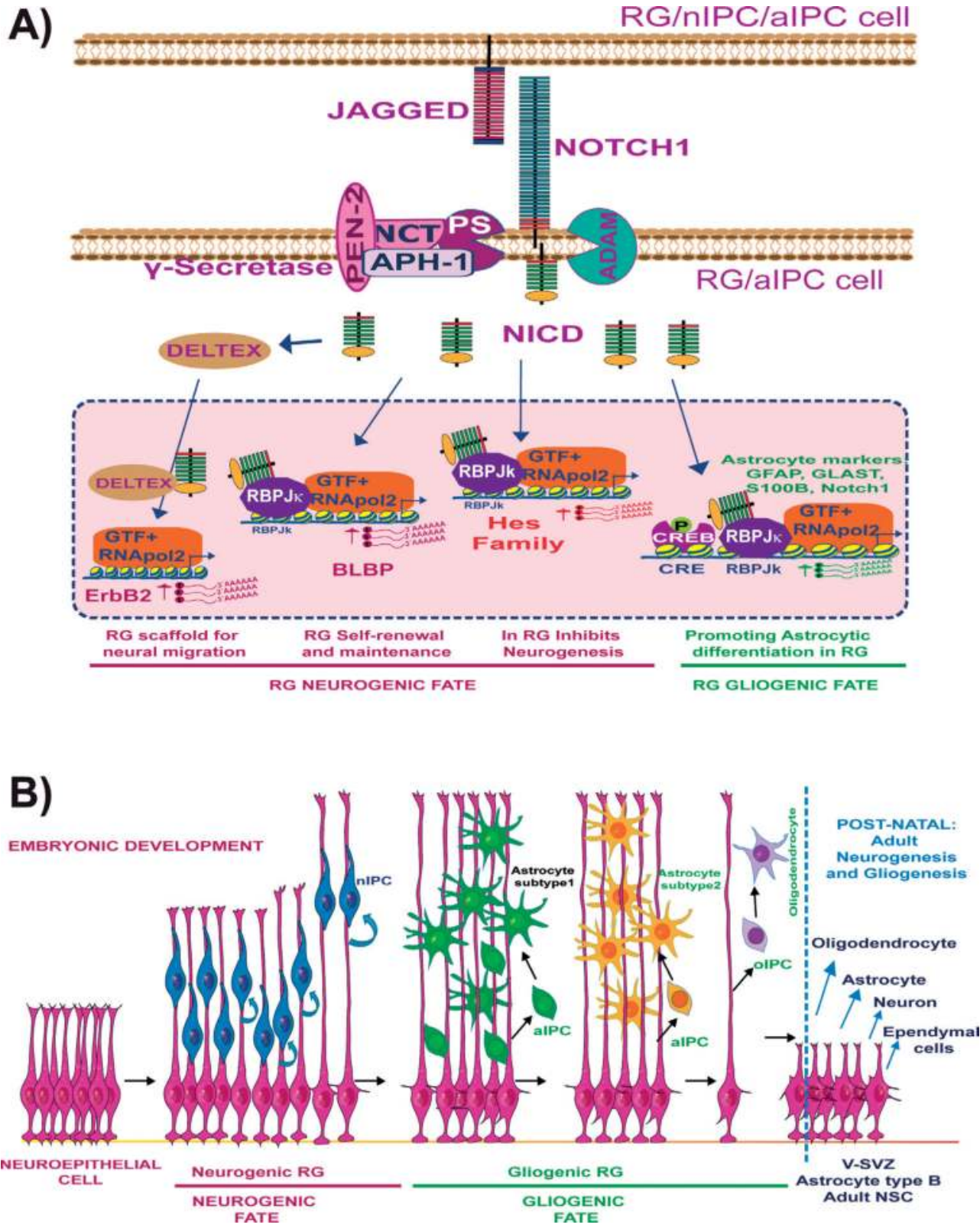

Figure 1. Notch pathway regulates astrocytic differentiation. (A) In timed cell genesis, a series of orchestrated events are activated to regulate cell differentiation; the Notch pathway is used as a signaling system during embryonic development and regulates different cell markers in astrocyte differentiation, such as Hes genes, GFAP and S100B. (nIPC/aIPC cell stands for neural or astrocytic intermediate precursor cell). (B) Neuroepithelial cells in early development proliferate by asymmetric cell division to generate more neuroepithelial cells (progenitor expansion phase). As brain development proceeds, neuroepithelial cells elongate to convert into radial glial (RG) cells and guide neuronal migration. Later, RG can divide asymmetrically to generate neuron, astrocyte, or oligodendrocyte intermediate progenitor cells. 
activate the cAMP-response element-binding protein (CREB) [42-48]. In a glial precursor-like model, C6 cells, cAMP-PKA-CREB activation leads to increase autocrine IL-6, which in turn activates STAT3, which induces GFAP promoter activation [49]. In the same model, the cAMPPKA-CREB signaling cascade cross talks with the Notch pathway, and together they act synergistically to reduce the progenitor marker (Nestin) and to induce astrocytic differentiation; measured by induced astrocytic markers (GFAP, S100 $\beta$, and GLAST) and glutamate uptake. In this context, Notch1 mRNA is up-regulated in a PKA/ $\gamma$-secretase/NICD/CSL-dependent manner, suggesting the establishment of a feedback loop to keep Notch pathway active until astrocytic differentiation is complete [50]. It is not surprising that the Notch pathway interacts with other signaling cascades to complete astrocytic differentiation. A bioinformatic analysis of promoters for GFAP, S100 $\beta$, GLAST, and Notch1, reveals specific sites for CREB, STAT3, and CSL transcription factors, suggesting that these three pathways, cAMP-PKA/JAK-STAT3/ Notch, cooperate to induce the transcription of astrocyte markers. Certainly, at this stage, other crosstalk interactions cannot be discarded.

\section{Glial differentiation and the $\mathrm{Ca}^{2+} /$ diacylglycerol-dependent protein kinase (PKC)}

As already mentioned, glial differentiation is modulated by extracellular signals, growth factors, hormones, cytokines, neurotrophins, and neurotransmitters that activate different signal pathways. PKC is one of the major mediators of these extracellular signals. The structure of this family of protein kinases contains a highly conserved catalytic domain and a regulatory domain (C1-C4 domains) responsible for its inactive conformation. Regulatory domains are separated by variable regions susceptible to proteolytic cleavage and essential for activation and conformational changes. The PKC family comprises 11 isoforms and is organized in three subfamilies: classical, novel, and atypical isoforms described in Table 2 [51-54].

PKC is highly expressed in the brain, with a significant role of this kinase in the function of neuronal and glial cells. The role of PKC in glial cells has been demonstrated in different reports, and PKC-activator PMA as well as the different PKC inhibitors, modify the cell morphology, proliferation, and differentiation [55-58]. Differential expression of PKC isoforms has been reported during neuronal development as four PKC isoforms are expressed in neuronal primary cultures of rat cerebellum. In contrast, only two isoforms, $\alpha$ PKC and $\beta I I$ PKC, are present in glial cultures $[59,60]$. Brodie et al. reported that in undifferentiated C6 cells, the PKC isoforms $\theta, \mu, \zeta$, and $\lambda$ are present; however, the cAMP-dependent differentiated C6 cells expressed significantly lower levels of PKC $\alpha$ and PKC $\delta$ and higher levels of PKC $\gamma, \eta$, and $\theta$.

Concerning PKC and glial cell function, overexpression of the $\beta$ and $\gamma$ isoforms increases GFAP levels, as a response to exposure to the PKC activator phorbol 12-myristate 13-acetate (PMA) treatment. Glutamine synthetase (GS) levels increase with PKC $\gamma$ overexpression and decrease with PKC $\delta$. Therefore, it is plausible that PKC $\alpha$ and $\delta$ provide negative signals for astrocytic differentiation, while PKC $\beta$ and $\gamma$ induce astrocyte differentiation [51].

However, it has also been documented that undifferentiated C6 cells express the $\alpha, \beta I I, \gamma, \delta, \varepsilon$, and $\zeta$ PKC isoforms and that long-term PKC inhibition after staurosporine treatment, which 


\begin{tabular}{lll}
\hline & Isoforms & Activity \\
\hline Classical isoforms & $\alpha, \beta I, \beta I I, \gamma$ & Dependent on DAG, PS, and $\mathrm{Ca}^{2+}$ \\
Novel PKC isoforms & $\delta, \varepsilon, \theta, \eta, \mu$ & Bind DAG, PS, and calcium-independent \\
Atypical PKC isoforms & $\iota / \lambda, \xi$ & Bind PIP3, calcium-independent and do not require DAG \\
\hline
\end{tabular}

Diacylglycerol (DAG), phosphatidylserine (PS), phosphatidylinositol 3-phosphate (PIP3) [53, 78].

Table 2. PKC classification.

leads to differentiation, results in $\beta$ II decrease, $\gamma$ increase and $\varepsilon$ translocation from the membrane to the cytosol [55]. Similar results were reported in the C6 cell differentiation process with dbcAMP [61]. It is clear that the molecular mechanisms triggered by glia differentiation agents are different, but that the various PKC isoforms are critically involved in the overall process.

In contrast, Watanabe et al. recently reported that overexpression of PKC $\beta$ II synergistically enhanced differentiation in the presence of $1 \mathrm{nM}$ of PACAP. These results indicate that the $\beta$ isoform of PKC is important in PACAP-induced differentiation of mouse embryonic NSCs into astrocytes via the PAC1 receptor, resulting in activation of phospholipase $\mathrm{C}$, followed by PKC activation. This latter observation was confirmed in NSCs. The cells were exposed to $2 \mathrm{nM}$ PACAP, resulting in a transient increase in the $\beta \mathrm{II}$ isoform, that returned to basal levels by day 4, whereas the levels of PKC $\alpha$ increased linearly up to day 6 [62].

RG cells and astrocytes are involved in regulation of the brain microenvironment, and glutamate transporters control the extracellular levels of this neurotransmitter. Regulation of glutamate uptake involves several factors like neuronal interactions, glutamate, CAMP, and phorbol esters. GLAST is the major glutamate transporter expressed in RG cells. Interestingly, GLAST expression is regulated via PKC through the reduction of its protein and mRNA levels. Our work group demonstrated that chglast transcriptional regulation is mediated by PKC, especially the $\alpha$ and $\varepsilon$ isoforms, which activate the AP- 1 transcription factor $[56,63,64]$.

\section{Another Notch in the belt: PKC/Notch cross talk in glial differentiation}

The Notch signaling pathway plays an important role in the control of cell fate during developmental processes. Several reports have shown that Notch-induced signaling interacts with other signaling pathways, such as NF- $\kappa \mathrm{B}$, the mitogen-activated protein kinase (MAPK) pathway, and the phosphatidylinositol 3-kinase (PI3K)/Akt pathway [65-67]. Although there are only a few reports describing the crosstalk between Notch and PKC signaling during glial differentiation, some connections have been described so far. The most direct example concerns the direct PKC action over NICD: Kim et al. found that PKC $\delta$ down-regulates NICD transcriptional activity in a kinase-independent manner. The mechanism involves the inhibition of the nuclear localization of NICD, most possibly through a physical association between NICD and PKC $\delta$ causing the dissociation of NICD from target gene promoters like Hes5 [67]. 
In another example, PMA increased the expression of Notch1 in a PKC $\varepsilon$-dependent manner in the context of astrocytic differentiation, and this is to say that in the course of PKCdependent astrocyte differentiation, an increase in Notch levels is found. In fact, serine 729 PKC $\varepsilon$ phosphorylation is as essential for the differentiation process. This data suggests that Notch1 is a plausible mediator of PKC $\varepsilon$ in astrocytic differentiation [68]. In the same line, $\mathrm{Xu}$ et al. reported that morphine-dependent astrocytic differentiation of neuronal progenitor cells (NPC) involves ERK via PKC $\varepsilon$ and TRBP phosphorylation that leads to miR-181a maturation, thus regulating the expression of Prox1 and Notch1 [69].

More recently, it has been demonstrated that atypical PKC isoforms participate in asymmetric cell division when glial differentiation starts. Sjoqvist et al. demonstrated that $\mathrm{PKC} \zeta$ regulates the Notch pathway by phosphorylation and regulation of Notch receptor traffic. When Notch signaling is active (after ligand stimulation or after expression of an activated membranetethered form of Notch), PKC $\zeta$ enhances the production of NICD and shifts the localization of Notch from late endosomes to the nucleus, leading to an elevated Notch signaling. In contrast, when the Notch receptor is not activated, $\mathrm{PKC} \zeta$ interacts with the receptor to induce a shift in receptor distribution from the plasma membrane to intracellular vesicles [70]. In C6 glioma cells, increased cAMP levels promote astrocytic commitment with a sustained augmentation of Notch activity, as detected by nuclear translocation of its intracellular domain portion (NICD) and its transcriptional activity [50]. The cAMP effect is mediated through the activation of the $\gamma$-secretase complex, responsible for Notch cleavage as demonstrated by its sensitivity to PKA inhibitors. As expected, Notch cleavage and nuclear translocation result in the upregulation of the mRNA levels of one of its target genes, the transcription factor Hair, and enhancer of split 5. Moreover, glutamate uptake activity, expression of astrocytic markers (genes responsible for glial progenitor cell fate decision) such as the glial fibrillary acidic protein, the S100beta protein, and GLAST, are also enhanced in cAMP-exposed cells [50]. Interestingly, polychlorinated biphenyls (PCBs) disturb the cAMP-induced astrocytic differentiation of C6 cells via the PKC isoforms $\gamma, \beta 2, \delta$ and $\varepsilon$ [58]. Additionally, PMA promotes adult neurogenesis by inducing neural progenitor cell proliferation in vitro in NPCs obtained from the SVZ of 7-day postnatal mice [71].

To support a plausible role of a crosstalk between PKC and Notch pathways in embryonic glial differentiation, we used chick Bergman radial glia from cerebellum (BGC) at day 14 of embryonic development and stimulated PKC using TPA. In this system, it was observed that PKC activation increased NICD/RPBJא-dependent transcription, measured by a reporter construct that senses directly the CSL activity (Figure 2A). This effect could be mediated by the classical PKC isoforms $(\alpha, \beta 1, \beta 2$, and $\gamma)$ and/or the novel isoforms ( $\delta$ and $\varepsilon)$, as it was observed when the specific inhibitor bisindolylmaleimide 1 (Bis1). The same effect was observed over a Hes1-responsive reporter (Figure 2A, right). Also, the MAPK/ERK pathway plays a role in PKC-mediated NICD/RPBJK activation, as demonstrated when specific MAPK/ERK inhibitors (U0126, PD98059, and SB202190) were used in co-treatment with TPA (Figure 2A). In contrast, treatment of BGC with TPA, down-regulates astrocytic biomarkers such as GFAP, GS, GLAST, FABP7, and Notch1 mRNA levels, and keeps Nestin, a progenitor marker, up-regulated (Figure 2B). Our results suggest that the activation of PKC induces NICD/RBPJK dependent transcriptional activation by a yet-to-be characterized mechanism, that perhaps 

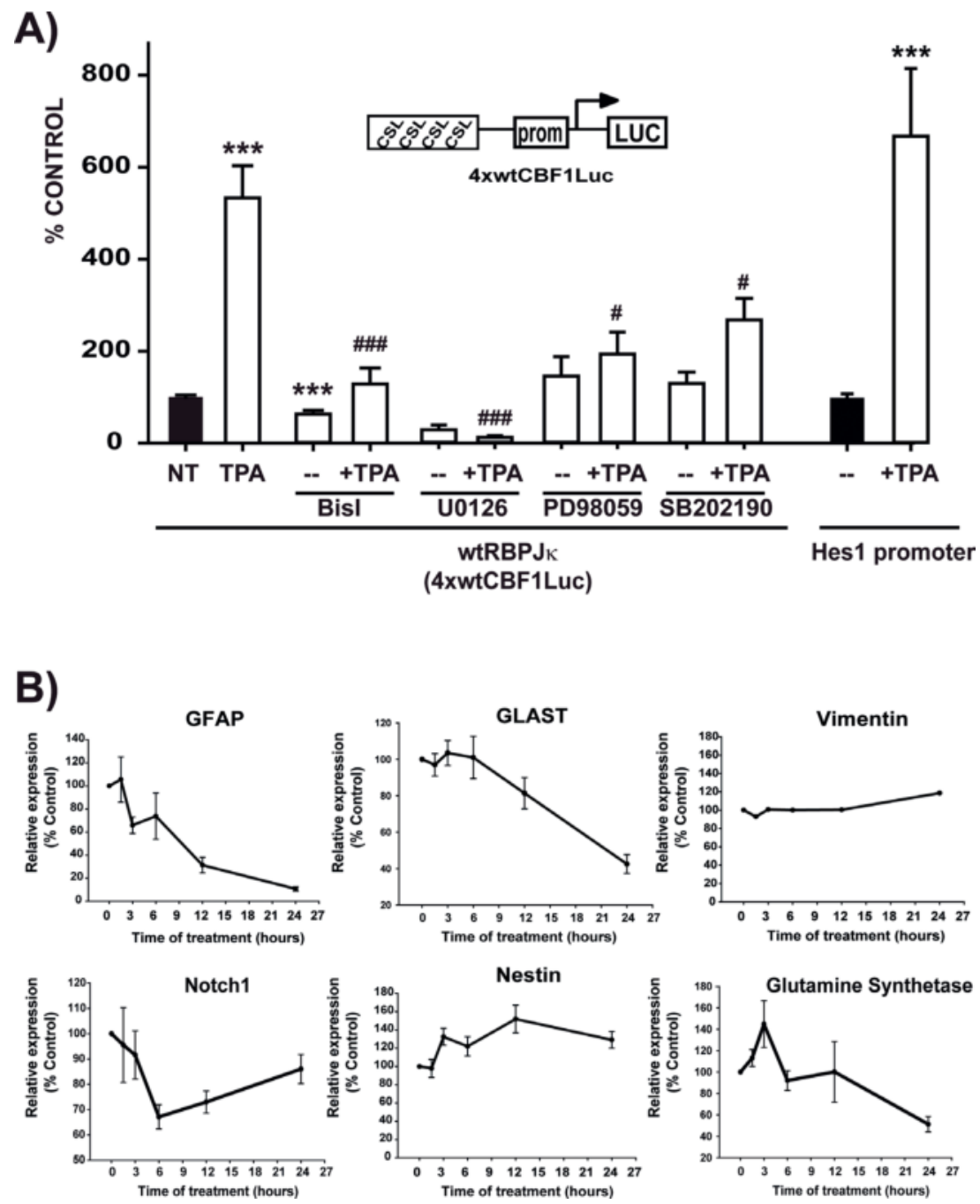

Figure 2. PKC/MAPK signaling activates Notch pathway in cultured Bergmann glia cells (BGCs). (A) In order to determine the role of the Notch/PKC signaling pathway in BGCs, cells (primary culture) were transfected with the reporter plasmid 4xwtCBF1Luc (containing four repeated sequences for RBPJк elements) or Hes1 promoter (a Notch effector gene) with Lipofectamine R 2000; after harvesting, the luciferase reporter activity was measured. $24 \mathrm{~h}$ post-transfection, the BGCs were treated with $100 \mathrm{nM}$ TPA for other $24 \mathrm{~h}$, where we showed that promoter activity increased five-fold in relation with nontreatment cells, and TPA induces the Notch signaling response. To analyze the signal pathway involved in Notch activation, BGCs were treated with $40 \mu \mathrm{M}$ BisI (a PKC inhibitor), $50 \mu \mathrm{M}$ U0126 (a MAPK1 inhibitor), PD (PD8059, a MAPK1 inhibitor), or SB20 (SB202190, a specific ERK inhibitor) $30 \mathrm{~min}$ prior to TPA, as indicated in the figure. Note that both PKC and MAPK inhibitors prevent Notch pathway activation. Results are presented as fold expression relative to nontreated cells. Data represent mean values \pm SE $(n=3)$. Data were analyzed by a one-way ANOVA with a post hoc Dunn's test. ${ }^{* * *} \mathrm{p}<0.001$; \#\# $\mathrm{p}<0.003$; \#, $\mathrm{p}<0.05$. (B) qRT-PCR was performed to analyze expression of Notch pathway targets, and BGC cultures were treated or not with TPA (100 nM TPA) at different times. Total RNA was extracted to amplify GFAP, GLAST, vimentin, glutamine synthetase (astrocytic markers), and nestin (radial glial marker) with the KAPA SYBR FAST one-step qRT-PCR kit. Our data suggest that TPA downregulates GLAST, GFAP, and GS and upregulates nestin; this evidence indicates that the Notch pathway is important in the radial glial fate. 
is related to regulation of NICD routing and trafficking $[67,70]$. The NICD/RBPJ $\kappa$ complex induces Hes1 expression, a well-known neurogenesis inhibitor. On the other hand, PKC activation blocks astrogenesis, perhaps modulating the access of NICD/RBPJ $\kappa$ to the astrocytic markers' promoters, like GFAP, which is dependent on Notch activation. In BGC, PKC activation regulates several genes that are closely related to glial function and induces radial glial phenotype as TPA down-regulates GLAST, GFAP, and GS and upregulates Nestin, PKC $\alpha$, or $\mathrm{PKC \varepsilon}(\mathrm{PKC} \odot 2)$.

\subsection{Notch pathway in CNS: some aspects of clinical relevance}

A plethora of pathological scenarios in the CNS are the result of neuronal degeneration. This cell loss needs to be compensated to keep the neural circuits working. In this context, neural stem cells can be differentiated into precise neuronal subtypes, but a common fact is that Notch signaling promotes astrocyte differentiation rather than neuronal differentiation. Therefore, Notch inhibition is an alternative therapeutic option in the clinical approach. Examples of the possible application of inhibiting Notch are presented in the Table 3.

\begin{tabular}{lllll}
\hline $\begin{array}{l}\text { Pathological context/ } \\
\text { biological system }\end{array}$ & $\begin{array}{l}\text { Targeted neural } \\
\text { stem cells }\end{array}$ & Notch signaling role and possible therapeutic approach & Ref \\
\hline
\end{tabular}

Blockage of notch pathway in neural adult stem cells to promote neurogenesis

Glioblastoma

(multiforme or grade IV

astrocytoma)
Glioblastoma cells (GB)
Lineage-specific differentiation of NPCs

\section{Alzheimer's disease} (AD)

Amyloid precursor protein (APP)

Blood-brain barrier, pathology

\begin{abstract}
Hippocampal progenitor cells (HPC)
\end{abstract}

Human neural progenitor cells (HNPC) inhibited tumor growth. Conversely, activation of Notch signaling promotes cell proliferation and colony formation in the human GB cell line. Notch1 promotes invasive migratory properties of GB cells by stimulating $\beta$-catenin and NF- $\mathrm{KB}$ signaling and mediates GB cell proliferation and survival through the Akt-mammalian target of rapamycin (mTOR) signaling axis. Treatment with $\gamma$-secretase inhibitors reduces neurosphere growth, and inhibits xenograft tumor growth through decreased Akt and STAT3 phosphorylation. Combination of Notch inhibitor MRK003 and Akt inhibitor MK-2206 effectively inhibited GB invasiveness.

Phosphorylation of TAR RNA-binding protein together with miR-181a maturation, as well as Dicer activity, is involved in morphine-induced astrocyte-preferential differentiation of HPC.

Activation of IL-6/gp130 and Notch signaling pathways in glial differentiation of HNPCs may cause problems in maintaining normal brain function and may contribute to AD pathology. Treatment with sAPP increased expression levels of GFAP in NT-2/D1 cells along with the generation of Notch intracellular domain (NICD) and expression of Hairy and enhancer of split 1 (Hes1), indicating that glial differentiation may aid in the development of novel therapeutic strategies for AD.

Brain endothelial cells

Neuron-derived Dll1 activates Notch signaling and is essentia for brain endothelial cells' survival as wells as blood-brain barrier, selective substance crossing; physiology, pathology, and drug development

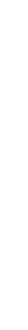




\begin{tabular}{|c|c|c|}
\hline $\begin{array}{l}\text { Pathological context/ } \\
\text { biological system }\end{array}$ & $\begin{array}{l}\text { Targeted neural } \\
\text { stem cells }\end{array}$ & Notch signaling role and possible therapeutic approach \\
\hline $\begin{array}{l}\text { Traumatic brain injury } \\
\text { (TBI), inflammation and } \\
\text { apoptosis and brain } \\
\text { edema }\end{array}$ & $\begin{array}{l}\text { Cerebral cortices } \\
\text { response }\end{array}$ & $\begin{array}{l}\text { Inhibition of Notch signaling by crocin, an extract of saffron, } \\
\text { has a neuroprotective effect against TBI, since in this type of } \\
\text { injury an upregulation of Notch intracellular domain (NICD) } \\
\text { and Hes } 1 \text { mRNA levels is present, decreasing microglial } \\
\text { activation and release of several pro-inflammatory cytokines. }\end{array}$ \\
\hline $\begin{array}{l}\text { Increased differentiation } \\
\text { of neural progenitor cells } \\
\text { when co-cultured with } \\
\text { astrocytes lacking glial } \\
\text { fibrillary acidic protein }\end{array}$ & $\begin{array}{l}\text { Neural progenitor } \\
\text { cells }\end{array}$ & $\begin{array}{l}\text { Astrocytes negatively regulate neurogenesis through Notch } \\
\text { pathway; endocytosis of Notch ligand Jagged } 1 \text { in astrocytes } \\
\text { and Notch signaling from astrocytes to neural stem/progenitor } \\
\text { cells depends on intermediate filament proteins GFAP and } \\
\text { vimentin. }\end{array}$ \\
\hline
\end{tabular}

Experimental autoimmune encephalomyelitis (failure to repair demyelination) as Multiple Sclerosis model (mice)

$\begin{array}{ll}\text { Seizure as a serious } & \text { Neurons in } \\ \text { complication of stroke } & \text { cortex and }\end{array}$
Oligodendrocyte precursor cells hippo-campus
Gamma-secretase inhibition of Notch signaling enhances tissue repair.

Notch pathway inhibits oligodendrocytes differentiation and hampers their ability to produce myelin during CNS development.

In a global cerebral ischemia model (GCI), there is augmented excitatory synaptic neurotransmission by upregulating glutamate receptor subunits (GluN2A, GluA1) and cotransporter $\mathrm{NKCC} 1$, but there is attenuated inhibitory synaptic neurotransmission by down-regulating amino butyric acid (GABA), and neuronal $\mathrm{K}-\mathrm{Cl}$ cotransporter. Aberrant activation of Notch signaling is involved in poststroke seizures, as NICD 1 and 2 were upregulated in the cerebral cortex and hippocampus post-GCI. DAPT treatment normalized the homeostasis of excitatory and inhibitory synaptic neurotransmission.

Leucine-rich repeat kinase 2 (LRRK2) complex promotes recycling of Notch ligand Delta-like 1 (Dll1)/Delta (D1) through modulation of endosomal trafficking and negatively regulates Notch signaling through cis-inhibition by stabilizing Dll1/Dl, accelerating neural stem cell differentiation; alteration of Notch signaling in mature neurons is a component of PD etiology linked to LRRK2.

Notch signaling in neurodegenerative diseases and pathological glutamate mediated plasticity

Dopamine release in the Neuronal cells striatum, individual's susceptibility to neuropsychiatric disease

Length, polarity, and synaptogenesis
Spiral ganglion neurons (SGNs)
RBP-J deficiency drastically reduced dopamine release in the striatum and caused a subtle decrease in the number of dopaminergic neurons as Notch/RBP-J signaling regulates dopamine responsiveness in the striatum.

DNER modulates length, polarity and synaptogenesis via the Notch signaling pathway. DNER was expressed in spiral ganglion neurons exhibiting significant polarity in early differentiation stages; DNER expression gradually decreased until polarity was lost on week 35 . Silencing DNER expression altered the polarity of differentiated neurons and these cells exhibited significantly reduced dendritic length.

Table 3. Notch signaling in cell therapy. 


\section{Conclusion}

Glia cells play an active role in glutamatergic transmission due to their compulsory intervention in the recycling of this excitatory neurotransmitter. The Notch signal transduction pathway is critically involved in the gene expression regulation of the major excitatory amino acid transporter expressed in early stages of astrocyte differentiation and in RG in the adult brain. Notch signaling involves the activation of diverse isoforms of PKC. Glial differentiation can be mediated by PKC and its isoforms, which act over NICD, increasing Notch expression, regulating several astrocytic markers related to glial function, and inducing the radial glial phenotype.

\section{Acknowledgements}

The work in our labs is funded by Conacyt-Mexico 255087 and Soluciones para un México Verde, S.A. de C.V. granted to AO and Conacyt-PEI 212650 and 231793 granted to ELB.

\section{Conflict of interest}

The authors declare that there are no conflicts of interest.

\section{Abbreviations}

ADAM10/TACE Tumor necrosis factor- $\alpha$ converting enzyme

AldhL1 aldehyde dehydrogenase 1

bFGF Basic fibroblast growth factor

BGC Chick Bergmann Radial glia

BisI Bisindolylmaleimide

BLBP Brain lipid-binding protein

BMP Bone morphogenetic proteins

cAMP Cyclic adenosine monophosphate

CIR Corepressor interacting with RBPJ 1

CNS Central nervous system

CNTF Ciliary neurotrophic factor 
CREB cAMP-response element-binding protein

CT-1 Cardiotrophin-1

DNMT1 DNA methyl transferase 1

DTX1 Deltex1

EAATs $\quad$ Excitatory amino acid transporters

EACC1 Excitatory amino acid transporter 1

EGF Epidermal growth factor

ErbB2 also known as HER2, Human epidermal growth factor receptor 2

ERK Extracellular signal-regulated kinases

FABP7 Fatty acid-binding protein 7

GFAP Glial fibrillary acidic protein

GLAST Glutamate aspartate transporter

GLT-1 Glutamate transporter 1

GS Glutamine synthetase

HDAC Histone deacetylases

LIF Leukemia inhibitory factor

LIFR $\beta \quad$ Leukemia inhibitory factor receptor $\beta$

MAM Mastermind

MAP 2 Microtubule-associated protein 2

MAPK Mitogen-activated protein kinase

MASH Mammalian achaete-scute homolog-1

NEXT Notch extracellular truncation

NFA1 Nuclear factor 1A

NF- $\kappa \mathrm{B} \quad$ Nuclear factor kappa-light-chain-enhancer of activated B cells

Ngn1 Neurogenin 1

NICD Notch intracellular domain

NIPC Intermediate progenitor cell 
PACAP Pituitary adenylate cyclase-activating polypeptide

PI3K Phosphatidylinositol 3-kinase

PKA cAMP-dependent protein kinase

PKC Protein kinase C

PMA Phorbol 12-myristate 13-acetate

RBPJא Recombining binding protein

RG Radial glia

SMRT Thyroid-hormone receptors

STAT3 Signal transducer and activator of transcription 3

SVZ Subventricular Zone

TN-C Tenascin C

TPA 12-O-tetradecanoylphorbol 13-acetate

TRBP TAR RNA-binding protein

\section{Author details}

López-Bayghen Esther ${ }^{1}$, Angulo-Rojo Carla², López-Bayghen Bruno ${ }^{1}$, Hernández-Melchor Dinorah ${ }^{1}$, Ramírez Leticia ${ }^{1}$ and Ortega Arturo $^{1 *}$

*Address all correspondence to: arortega@cinvestav.mx

1 Departamento de Toxicología, Centro de Investigación de Estudios Avanzados del IPN, México City, Mexico

2 Unidad de Investigación, Facultad de Medicina, Universidad Autónoma de Sinaloa, Culiacán, Sinaloa, Mexico

\section{References}

[1] Gasic GP et al. Molecular neurobiology of glutamate receptors. Annual Review of Physiology. 1992;54:507-536. DOI: 10.1146/annurev.ph.54.030192.002451

[2] Hollmann M et al. Cloned glutamate receptors. Annual Review of Neuroscience. 1994;17: 31-108. DOI: 10.1146/annurev.ne.17.030194.000335 
[3] Schoepp DD et al. Metabotropic glutamate receptors and neuronal degenerative disorders. Neurobiology of Aging. 1994;15(2):261-263

[4] Pin JP et al. The metabotropic glutamate receptors: Structure and functions. Neuropharmacology. 1995;34(1):1-26

[5] Cameron RS et al. Glial cell lineage in the cerebral cortex: A review and synthesis. Glia. 1991;4(2):124-137. DOI: 10.1002/glia.440040204

[6] Malatesta P et al. Neuronal or glial progeny: Regional differences in radial glia fate. Neuron. 2003;37(5):751-764

[7] Anthony TE et al. Radial glia serve as neuronal progenitors in all regions of the central nervous system. Neuron. 2004;41(6):881-890

[8] Danbolt NC. Glutamate uptake. Progress in Neurobiology. 2001;65(1):1-105

[9] Eulenburg V et al. Neurotransmitter transporters expressed in glial cells as regulators of synapse function. Brain Research Reviews. 2010;63(1-2):103-112. DOI: 10.1016/j. brainresrev.2010.01.003

[10] Swanson CJ et al. Metabotropic glutamate receptors as novel targets for anxiety and stress disorders. Nature Reviews. Drug Discovery. 2005;4(2):131-144. DOI: 10.1038/nrd1630

[11] Maragakis NJ et al. Glutamate transporter expression and function in human glial progenitors. Glia. 2004;45(2):133-143. DOI: 10.1002/glia.10310

[12] Martinez-Lozada Z et al. Glutamatergic transmission: A matter of three. Neural Plasticity. 2015;2015:787396. DOI: 10.1155/2015/787396

[13] Araque A et al. Glial modulation of synaptic transmission in culture. Glia. 2004;47(3):241248. DOI: 10.1002/glia.20026

[14] Gonzalez MI et al. Regulation of high-affinity glutamate uptake activity in Bergmann glia cells by glutamate. Brain Research. 2000;866(1-2):73-81

[15] Gegelashvili M et al. Glutamate transporter Glast/Eaat1 directs cell surface expression of Fxyd2/gamma subunit of $\mathrm{Na}$, K-Atpase in human fetal astrocytes. Neurochemistry International. 2007;50(7-8):916-920. DOI: 10.1016/j.neuint.2006.12.015

[16] Rose EM et al. Glutamate transporter coupling to Na,K-Atpase. The Journal of Neuroscience. 2009;29(25):8143-8155. DOI: 10.1523/JNEUROSCI.1081-09.2009

[17] Martinez-Lozada Z et al. Glast/Eaat1-induced glutamine release via Snat3 in Bergmann glial cells: Evidence of a functional and physical coupling. Journal of Neurochemistry. 2013;125(4):545-554. DOI: 10.1111/jnc.12211

[18] Danbolt NC et al. Neuronal vs glial glutamate uptake: Resolving the conundrum. Neurochemistry International. 2016;98:29-45. DOI: 10.1016/j.neuint.2016.05.009

[19] Flores-Mendez M et al. Glia plasma membrane transporters: Key players in Glutamatergic neurotransmission. Neurochemistry International. 2016;98:46-55. DOI: 10.1016/j. neuint.2016.04.004 
[20] Fortini ME. Notch and presenilin: A proteolytic mechanism emerges. Current Opinion in Cell Biology. 2001;13(5):627-634. DOI: 10.1016/S0955-0674(00)00261-1

[21] Fortini ME. Gamma-secretase-mediated proteolysis in cell-surface-receptor signalling. Nature Reviews. Molecular Cell Biology. 2002;3(9):673-684. DOI: 10.1038/nrm910

[22] Fortini ME et al. The suppressor of hairless protein participates in notch receptor signaling. Cell. 1994;79(2):273-282. DOI: 10.1016/0092-8674(94)90196-1

[23] Kopan $\mathrm{R}$ et al. The canonical notch signaling pathway: Unfolding the activation mechanism. Cell. 2009;137(2):216-233. DOI: 10.1016/j.cell.2009.03.045

[24] Bray SJ. Notch signalling: A simple pathway becomes complex. Nature Reviews. Molecular Cell Biology. 2006;7(9):678-689. DOI: 10.1038/nrm2009

[25] Chiba S. Concise review: Notch signaling in stem cell systems. Stem Cells. 2006;24(11): 2437-2447. DOI: 10.1634/stemcells.2005-0661

[26] Selkoe D et al. Notch and presenilin: Regulated intramembrane proteolysis links development and degeneration. Annual Review of Neuroscience. 2003;26(1):565-597. DOI: 10.1146/ annurev.neuro.26.041002.131334

[27] Mumm JS et al. Notch signaling: From the outside in. Developmental Biology. 2000; 228(2):151-165. DOI: 10.1006/dbio.2000.9960

[28] Gorman MJ et al. A genetic analysis of Deltex and its interaction with the notch locus in drosophila melanogaster. Genetics. 1992;131(1):99-112

[29] Matsuno K et al. Deltex acts as a positive regulator of notch signaling through interactions with the notch Ankyrin repeats. Development. 1995;121(8):2633-2644

[30] Yamamoto $N$ et al. Role of Deltex-1 as a transcriptional regulator downstream of the notch receptor. The Journal of Biological Chemistry. 2001;276(48):45031-45040. DOI: 10.1074/jbc.M105245200

[31] Kriegstein A et al. The glial nature of embryonic and adult neural stem cells. Annual Review of Neuroscience. 2009;32:149-184. DOI: 10.1146/annurev.neuro.051508.135600

[32] Patten BA et al. Sequential signaling through Notch1 and Erbb receptors mediates radial glia differentiation. The Journal of Neuroscience. 2003;23(14):6132-6140 23/14/6132 [pii]

[33] Patten BA et al. Notch1 signaling regulates radial glia differentiation through multiple transcriptional mechanisms. The Journal of Neuroscience. 2006;26(12):3102-3108. DOI: 10.1523/JNEUROSCI.4829-05.2006

[34] Aguirre A et al. Postnatal neurogenesis and gliogenesis in the olfactory bulb from Ng2-expressing progenitors of the subventricular zone. The Journal of Neuroscience. 2004;24(46):10530-10541. DOI: 10.1523/jneurosci.3572-04.2004

[35] Spassky N et al. Adult ependymal cells are postmitotic and are derived from radial glial cells during embryogenesis. The Journal of Neuroscience. 2005;25(1):10-18. DOI: 10.1523/jneurosci.1108-04.2005 
[36] Wang DD et al. The astrocyte odyssey. Progress in Neurobiology. 2008;86(4):342-367. DOI: 10.1016/j.pneurobio.2008.09.015

[37] Liu X et al. Gfap-expressing cells in the postnatal subventricular zone display a unique glial phenotype intermediate between radial glia and astrocytes. Glia. 2006;54(5):394410. DOI: $10.1002 /$ glia.20392

[38] Kimelberg HK. The problem of astrocyte identity. Neurochemistry International. 2004; 45(2-3):191-202. DOI: 10.1016/j.neuint.2003.08.015

[39] Cahoy JD et al. A transcriptome database for astrocytes, neurons, and oligodendrocytes: A new resource for understanding brain development and function. The Journal of Neuroscience. 2008;28(1):264-278. DOI: 10.1523/jneurosci.4178-07.2008

[40] Wilczynska KM et al. Nuclear factor I isoforms regulate gene expression during the differentiation of human neural progenitors to astrocytes. Stem Cells. 2009;27(5):1173-1181. DOI: $10.1002 /$ stem.35

[41] Cebolla B et al. Nuclear factor-I regulates glial fibrillary acidic protein gene expression in astrocytes differentiated from cortical precursor cells. Journal of Neurochemistry. 2006;97(4):1057-1070. DOI: 10.1111/j.1471-4159.2006.03804.x

[42] Vallejo I et al. Pituitary adenylate cyclase-activating polypeptide induces astrocyte differentiation of precursor cells from developing cerebral cortex. Molecular and Cellular Neurosciences. 2002;21(4):671-683. DOI: 10.1006/mcne.2002.1189

[43] Jozwiak-Bebenista M et al. Effects of Pacap, Vip and related peptides on cyclic amp formation in rat neuronal and astrocyte cultures and cerebral cortical slices. Pharmacological Reports. 2007;59(4):414-420. DOI: 10.1016/j.peptides.2007.04.007

[44] Vallejo M. Pacap signaling to dream: A camp-dependent pathway that regulates cortical Astrogliogenesis. Molecular Neurobiology. 2009;39(2):90-100. DOI: 10.1007/s12035009-8055-2

[45] Tatsuno I et al. Pituitary adenylate cyclase-activating polypeptide (Pacap) is a regulator of astrocytes: Pacap stimulates proliferation and production of interleukin 6 (Il-6), but not nerve growth factor (Ngf), in cultured rat astrocyte. Annals of the New York Academy of Sciences. 1996;805:482-488. DOI: 10.1111/j.1749-6632.1996.tb17508.x

[46] Nakamachi T et al. Role of Pacap in neural stem/progenitor cell and astrocyte: From neural development to neural repair. Current Pharmaceutical Design. 2011;17(10):973-984. DOI: $10.2174 / 138161211795589346$

[47] Nakamachi T et al. Pituitary adenylate cyclase-activating polypeptide (Pacap) type 1 receptor (Pac1r) co-localizes with activity-dependent Neuroprotective protein (Adnp) in the mouse brains. Regulatory Peptides. 2008;145(1):88-95. DOI: 10.1016/j.regpep.2007.09.025

[48] Sands WA et al. Regulating gene transcription in response to cyclic amp elevation. Cellular Signalling. 2008;20(3):460-466. DOI: 10.1016/j.cellsig.2007.10.005 
[49] Takanaga H et al. Camp-induced astrocytic differentiation of C6 Glioma cells is mediated by Autocrine Interleukin-6. The Journal of Biological Chemistry. 2004;279(15):1544115447. DOI: $10.1074 / j b c . M 311844200$

[50] Angulo-Rojo C et al. Involvement of the notch pathway in terminal astrocytic differentiation: Role of Pka. ASN Neuro. 2013;5(5):e00130. DOI: 10.1042/AN20130023

[51] Brodie C et al. Differential role of specific Pkc isoforms in the proliferation of glial cells and the expression of the astrocytic markers Gfap and glutamine Synthetase. Molecular Brain Research. 1998;56(1):108-117. DOI: 10.1016/S0169-328X(98)00035-7

[52] Nishizuka Y. Protein kinase $C$ and lipid signaling for sustained cellular responses. The FASEB Journal. 1995;9(7):484-496

[53] Newton AC. Protein kinase C: Structure, function, and regulation. The Journal of Biological Chemistry. 1995;270(48):28495-28498. DOI: 10.1074/jbc.270.48.28495

[54] do Carmo A et al. Pkc signaling in glioblastoma. Cancer Biology \& Therapy. 2013;14(4): 287-294. Doi: $10.4161 /$ cbt.23615

[55] Kronfeld I et al. Staurosporine induces astrocytic phenotypes and differential expression of specific Pkc isoforms in C6 glial cells. Journal of Neurochemistry. 1995;65(4):15051514. DOI: 10.1046/j.1471-4159.1995.65041505.x

[56] Lopez-Bayghen E et al. Glutamate-dependent transcriptional regulation of Glast: Role of Pkc. Journal of Neurochemistry.2004;91(1):200-209.DOI:10.1111/j.1471-4159.2004.02706.x

[57] Moreton $\mathrm{K}$ et al. Protein expression of the $\mathrm{A}, \Gamma, \Delta$ and $\mathrm{E}$ subspecies of protein kinase $\mathrm{C}$ changes as C6 Glioma cells become contact inhibited and quiescent in the presence of serum. FEBS Letters. 1995;372(1):33-38 https://doi.org/10.1016/0014-5793(95)00941-2

[58] Adornetto A et al. Polychlorinated biphenyls impair Dibutyryl camp-induced astrocytic differentiation in rat C6 glial cell line. FEBS Open Bio. 2013;3:459-466. DOI: 10.1016/j. fob.2013.10.008

[59] Masliah E et al. Differential involvement of protein kinase C Isozymes in Alzheimer's disease. The Journal of Neuroscience. 1990;10(7):2113-2124

[60] Moreton $\mathrm{K}$ et al. Changes in Pkc subspecies protein expression as C6 cells reach $\mathrm{G}(0)$ by contact inhibition in the presence of serum. Biochemical Society Transactions. 1995; 23(3):446S)

[61] S-i Y et al. Differential expression of rho family Gtp-binding proteins and protein kinase C Isozymes during C6 glial cell differentiation. Molecular Brain Research. 1997;45(1): 90-98. DOI: 10.1016/S0169-328X(96)00239-2

[62] Watanabe J et al. Pituitary adenylate cyclase-activating polypeptide-induced differentiation of embryonic neural stem cells into astrocytes is mediated via the Beta isoform of 
protein kinase C. Journal of Neuroscience Research. 2006;84(8):1645-1655. DOI: 10.1002/ jnr.21065

[63] Bernabe A et al. Regulation of the Na+-dependent glutamate/aspartate transporter in rodent cerebellar astrocytes. Neurochemical Research. 2003;28(12):1843-1849 https://doi. org/10.1023/A\%3A1026171724963

[64] Espinoza-Rojo M et al. Glast: Gene expression regulation by Phorbol esters. Neuroreport. 2000;11(12):2827-2832

[65] Guo D et al. Notch-1 regulates Akt signaling pathway and the expression of cell cycle regulatory proteins Cyclin D1, Cdk2 and P21 in T-all cell lines. Leukemia Research. 2009; 33(5):678-685. DOI: 10.1016/j.leukres.2008.10.026

[66] Fitzgerald $\mathrm{K}$ et al. Ras pathway signals are required for notch-mediated oncogenesis. Oncogene. 2000;19(37):4191-4198. DOI: 10.1038/sj.onc.1203766

[67] Kim M et al. Protein kinase Cdelta negatively regulates Notch1-dependent transcription via a kinase-independent mechanism in vitro. Biochimica et Biophysica Acta. 2012;1823(2):387-397. DOI: 10.1016/j.bbamcr.2011.11.005

[68] Steinhart R et al. Pkcepsilon induces astrocytic differentiation of multipotential neural precursor cells. Glia. 2007;55(2):224-232. DOI: 10.1002/glia.20454

[69] Xu C et al. Morphine promotes astrocyte-preferential differentiation of mouse hippocampal progenitor cells via Pkcepsilon-dependent Erk activation and Trbp phosphorylation. Stem Cells. 2015;33(9):2762-2772. DOI: 10.1002/stem.2055

[70] Sjoqvist $\mathrm{M}$ et al. Pkczeta regulates notch receptor routing and activity in a notch signaling-dependent manner. Cell Research. 2014;24(4):433-450. DOI: 10.1038/cr.2014.34

[71] Guillem AM et al. Methylphenidate increases glutamate uptake in Bergmann glial cells. Neurochemical Research. 2015;40(11):2317-2324. DOI: 10.1007/s11064-015-1721-z

[72] Ohtsuka T et al. Hes1 and Hes5 as notch effectors in mammalian neuronal differentiation. The EMBO Journal. 1999;18(8):2196-2207. DOI: 10.1093/emboj/18.8.2196

[73] Lebkuechner I et al. Heterogeneity of notch signaling in astrocytes and the effects of Gfap and Vimentin deficiency. Journal of Neurochemistry. 2015;135(2):234-248. DOI: 10.1111/jnc.13213

[74] Endo $\mathrm{Y}$ et al. Bimodal functions of notch-mediated signaling are involved in neural crest formation during avian ectoderm development. Development. 2002;129(4):863-873

[75] Kwon C et al. Notch post-translationally regulates beta-catenin protein in stem and progenitor cells. Nature Cell Biology. 2011;13(10):1244-1251. DOI: 10.1038/ncb2313

[76] Ramain $P$ et al. Novel notch alleles reveal a Deltex-dependent pathway repressing neural fate. Current Biology. 2001;11(22):1729-1738 https://doi.org/10.1016/S0960-9822 (01)00562-0

[77] Endo $\mathrm{Y}$ et al. Deltex/Dtx mediates notch signaling in regulation of Bmp4 expression in cranial neural crest formation during avian development. Development, Growth \& Differentiation. 2003;45(3):241-248. DOI: 10.1046/j.1524-4725.2003.693.x 
[78] Kang JH et al. Protein kinase C $(\mathrm{Pkc})$ isozyme-specific substrates and their design. Biotechnology Advances. 2012;30(6):1662-1672. DOI: 10.1016/j.biotechadv.2012.07.004

[79] Wu S et al. Targeting Glioma stem cells for therapy: Perspectives and challenges. Journal of Cell Science and Therapy. 2015;6(207). DOI: 10.4172/2157-7013.1000207

[80] Sugaya K. Stem cell biology in the study of pathological conditions. Neurodegenerative Diseases. 2010;7(1-3):84-87. DOI: 10.1159/000285512

[81] Kwak Y-D et al. Involvement of notch signaling pathway in amyloid precursor protein induced glial differentiation. European Journal of Pharmacology. 2011;650(1):18-27. DOI: 10.1016/j.ejphar.2010.09.015

[82] Yamamizu $\mathrm{K}$ et al. In vitro modeling of blood-brain barrier with human Ipsc-derived endothelial cells, pericytes, neurons, and astrocytes via notch signaling. Stem Cell Reports. 2017;8(3):634-647. DOI: 10.1016/j.stemcr.2017.01.023

[83] Wang $\mathrm{K}$ et al. Neuroprotective effects of Crocin against traumatic brain injury in mice: Involvement of notch signaling pathway. Neuroscience Letters. 2015;591:53-58. DOI: 10.1016/j.neulet.2015.02.016

[84] Wilhelmsson U et al. Astrocytes negatively regulate neurogenesis through the Jagged1mediated notch pathway. Stem Cells. 2012;30(10):2320-2329. DOI: 10.1002/stem.1196

[85] Jurynczyk M et al. Inhibition of notch signaling enhances tissue repair in an animal model of multiple sclerosis. Journal of Neuroimmunology. 2005;170(1-2):3-10. DOI: 10.1016/j.jneuroim.2005.10.013

[86] Jurynczyk M et al. Overcoming failure to repair demyelination in Eae: Gamma-secretase inhibition of notch signaling. Journal of the Neurological Sciences. 2008;265(1-2):5-11. DOI: $10.1016 /$ j.jns.2007.09.007

[87] Yang GS et al. Synergistic effect of mild hypothermia and the notch inhibitor Dapt against post stroke seizures. Biomedicine \& Pharmacotherapy. 2017;96:675-684. DOI: 10.1016/j.biopha.2017.10.036

[88] Andersson ER et al. Therapeutic modulation of notch Signalling - Are we there yet? Nature Reviews Drug Discovery. 2014;13:357. DOI: 10.1038/nrd4252 https://www.nature. com/articles/nrd4252\#supplementary-information

[89] Toritsuka M et al. Regulation of striatal dopamine responsiveness by notch/Rbp-J signaling. Translational Psychiatry. 2017;7:e1049. DOI: 10.1038/tp.2017.21 https://www.nature. com/articles/tp201721\#supplementary-information

[90] Du J et al. Dner modulates the length, polarity and synaptogenesis of spiral ganglion neurons via the notch signaling pathway. Molecular Medicine Reports. 2017;17:2357-2365. DOI: $10.3892 / \mathrm{mmr} .2017 .8115$ 
